\title{
Understanding the diverse functions of Huatan Tongluo Fang on rheumatoid arthritis from a pharmacological perspective
}

\author{
CHUNSONG ZHENG ${ }^{1}$, MINGSHAN QIU ${ }^{2}$, XIAOJIE XU ${ }^{1,3}$, HONGZHI YE $^{1,4}$, \\ QIAN ZHANG ${ }^{2}$, YIHAN LI ${ }^{2}$, XIANXIANG LIU ${ }^{1}$ and JINCHUN CHEN ${ }^{2}$ \\ ${ }^{1}$ Institute of Bone Disease, Academy of Integrative Medicine, Fujian University of Traditional Chinese Medicine, \\ Fuzhou, Fujian 350122; ${ }^{2}$ Department of Rheumatism and Immunology, \\ Affiliated Xiamen Hospital of Traditional Chinese Medicine, Fujian University of Traditional Chinese Medicine, \\ Xiamen, Fujian 361009; ${ }^{3}$ College of Chemistry and Molecular Engineering, Peking University, Beijing 100871; \\ ${ }^{4}$ Fujian Key Laboratory of Integrative Medicine on Geriatrics, Fujian University of Traditional Chinese Medicine, \\ Fuzhou, Fujian 350122, P.R. China
}

Received January 26, 2015; Accepted March 3, 2016

DOI: $10.3892 /$ etm.2016.3329

\begin{abstract}
Huatan Tongluo Fang (HTTLF) is a traditional herbal formula that can resolve phlegm and dredge collaterals. HTTLF has also been used to treat rheumatoid arthritis (RA); however, the mechanism underlying the therapeutic effects of HTTLF on RA has not been clearly elucidated at the molecular level. In the present study, an integrated model of system pharmacology containing chemical space analysis, potential active compound prediction and compound-target-disease network was constructed to investigate the molecular mechanisms of HTTLF. The compounds from HTTLF dispersed well in the chemical space. Most of the compounds from HTTLF had similar chemical spaces to drug/drug-like compounds
\end{abstract}

Correspondence to: Professor Jinchun Chen, Department of Rheumatism and Immunology, Affiliated Xiamen Hospital of Traditional Chinese Medicine, Fujian University of Traditional Chinese Medicine, 1739 Xianyue Road, Xiamen, Fujian 361009, P.R. China

E-mail: chenjinchunfjtcm@163.com

Abbreviations: RA, rheumatoid arthritis; TCMs, traditional Chinese medicines; HTTLF, Huatan Tongluo Fang; BA, Bile arisaema; SP, Semen persicae; FC, Flos carthami; SA, Sinapis alba; BB, Bombyx batryticatus; PA, Paeonia alba; CIA, collagen-induced arthritis; VEGF, vascular endothelial growth factor; MMP, matrix metalloproteinase; MMFF, Merck molecular force field; MW, molecular weight; nHDon, number of hydrogen bond donors; nHAcc, number of hydrogen bond acceptors; AlogP, octanol-water partition coefficients; nRB, number of rotatable bonds; MPSA, molecular polar surface area; PDB, Protein Data Bank; COX-2, cyclooxygenase-2; JAKs, janus kinases; TNF- $\alpha$, tumor necrosis factor $\alpha$; Syk, spleen tyrosine kinase; MAP, mitogen-activated protein; Std Dev, standard deviation; TTD, Therapeutic Targets Database; QSAR, quantitative structure-activity relationship

Key words: rheumatoid arthritis, systems pharmacology, Huatan Tongluo Fang, traditional Chinese medicine, diverse functions associated with RA, according to the MDL Drug Data Report. A total of 127 potentially active compounds and 17 targets of RA were identified. Among them, 50 compounds interacted with $\geq 2$ targets, while 77 compounds interacted with only one target. In addition, 17 targets were associated with 82 diseases that belonged to 26 categories. These results indicate that HTTLF has diverse chemical spaces and polypharmacology with regards to the treatment of RA. In addition, HTTLF demonstrated therapeutic potential against diverse diseases other than RA, including osteoarthritis, atherosclerosis and brain cancer. This study provides a novel platform for understanding how HTTLF treats RA; this is beneficial for explaining the diverse functions of HTTLF with regards to RA, and may help develop novel compounds with desirable therapeutic targets to treat RA.

\section{Introduction}

Rheumatoid arthritis (RA) is an autoimmune disease primarily characterized by arthrosynovitis (1). Its main clinical manifestations are chronic, symmetrical, multi-joint synovitis and articular damage (2). The incidence of RA is $\sim 1 \%$ worldwide; RA severely influences quality of life and health as it can result in a high level of disability, and negatively affects individuals, families and society (3). At present, there is no individually recognized drug to control and treat RA. The primary drugs on the market for RA treatment are non-steroidal anti-inflammatory drugs, biological agents, disease-modifying anti-rheumatic drugs and glucocorticoids. However, the pharmacological management of RA has targeted the symptoms of the disease, rather than the underlying causes(4). In addition, the prolonged use of these drugs has numerous side-effects, and they are becoming ineffective as a result of drug resistance (5). Thus, it is important that researchers develop novel anti-rheumatic drugs that delay the progression of RA and reduce disability.

Traditional Chinese medicines (TCMs) have been used to treat RA for $>2,000$ years. It has been demonstrated that 
the effect of herbal formulas on RA is an integrated result of various mechanisms of action, including immunity adjustment and inflammatory control (6). Herbal formulae serve a moderate role in the treatment of RA; they have few side-effects and are suitable for long-term use $(7,8)$. The herbal treatment of RA has received increasing attention (9); it is thought that they have great potential to be developed and utilized for the treatment of patients with RA.

Huatan Tongluo Fang (HTTLF) is a traditional herbal formula that has been widely prescribed to treat RA in the Xiamen Hospital of Traditional Chinese Medicine (Xiamen, China). HTTLF is composed of six herbs, including Bile arisaema (BA; Dannanxing), Semen persicae (SP; Taoren), Flos carthami (FC; Honghua), Sinapis alba (SA; Baijiezi), Bombyx batryticatus (BB; Jiangcan) and Paeonia alba (PA; Baishao). Clinical observations have demonstrated that HTTLF can reduce the level of vascular endothelial growth factor (VEGF) in the serum of patients with RA, and significantly alleviate the indexes of erythrocyte rate, C-reactive protein, tenderness and swelling of the joints of patients with RA (10). The results of animal model experiments have demonstrated that HTTLF can relieve inflammation in rats with collagen-induced arthritis, and significantly reduce the expression levels of serum VEGF and matrix metalloproteinase (MMP)-3 (11). However, the underlying molecular mechanisms of HTTLF remain unknown. Fortunately, numerous computer simulation methods have made a significant contribution towards understanding the theory of TCMs and their mechanisms of action at a molecular and systems level (12-14). In the present study, an integrated model of systems pharmacology, developed in a previous study $(12,13)$, that combined molecular database building, chemical space, molecular docking and network pharmacological techniques, was used to investigate the molecular characteristics of HTTLF and map a compound-target-disease network to understand the interaction between HTTLF and therapeutic targets of RA from a systematic point of view. These attempts may offer novel opportunities to investigate the pharmacological basis of HTTLF, and provide an effective method to aid development of treatments for RA using herbal formulae.

\section{Materials and methods}

Molecular database building. All chemical ingredients from the six herbs of HTTLF were collected from the Chinese Herbal Drug Database, the Handbook of the Constituents in Chinese Herb Original Plants and other literature (15-19). A total of 692 compounds were obtained, of which 144 were obtained from Dannanxing, 68 from Taoren, 163 from Honghua, 119 from Baijiezi, 93 from Jiangcan and 105 from Baoshao. The chemical structures were drawn using ISIS Draw version 2.5 (MDL Information Systems, Inc., San Leandro, CA, USA) and further optimized by Discovery Studio version 2.0 (DS 2.0; Accelrys, Ltd., San Diego, CA, USA) with a Merck molecular force field (MMFF). In addition, 1,362 RA-associated drug/drug-like compounds were collected from the MDL Drug Data Report (20); these were optimized with the MMFF and saved to files in standard definition format in preparation for the subsequent analyses (21).
Table I. 17 key protein targets associated with rheumatoid arthritis.

Protein name

PDB code

$\begin{array}{lc}\text { Dihydroorotate dehydrogenase, mitochondrial } & 3 \mathrm{O} 8 \mathrm{~A} \\ \text { Cyclooxygenase-2 } & 3 \mathrm{MQE} \\ \text { Tyrosine-protein kinase Janus kinase } 3 & 3 \mathrm{LXL} \\ \text { Tumor necrosis factor- } \alpha & 2 \mathrm{AZ5} \\ \text { Interleukin } 1 \text { receptor } & 1 \mathrm{IRA} \\ \text { Integrin } \alpha-4 & 3 \mathrm{~V} 4 \mathrm{~V} \\ \text { Thioredoxin reductase, cytoplasmic } & 4 \mathrm{~B} 1 \mathrm{~B} \\ \text { Interleukin-2 } & 1 \mathrm{M} 48 \\ \text { Cathepsin K } & 1 \mathrm{AU} 0 \\ \text { Janus kinase 1 } & 4 \mathrm{IVD} \\ \text { Tyrosine-protein kinase SYK } & 4 \mathrm{FZ6} \\ \text { Mitogen-activated kinase p38 } & 1 \mathrm{CM} 8 \\ \text { Metalloproteinase domain-17 } & 2 \mathrm{~A} 8 \mathrm{H} \\ \text { Inhibitor of nuclear factor } \kappa \mathrm{B} \text { kinase } & 3 \mathrm{RZF} \\ \text { Matrix metalloproteinase- } 9 & 1 \mathrm{GKC} \\ \text { Vascular endothelial growth factor receptor } 2 & 1 \mathrm{Y} 6 \mathrm{~A} \\ \text { Macrophage migration inhibitory factor } & 4 \mathrm{~F} 2 \mathrm{~K}\end{array}$

PDB, Protein Data Bank; SYK, spleen tyrosine kinase.

Chemical space analysis. In the current study, a total of 150 physicochemical properties were calculated by the quantitative structure-activity relationship (QSAR) module of DS 2.0 (13), and principal components analysis was used to map the distributions of HTTLF and drug/drug-like compounds in the chemical space in two dimensions. According to Lipinski's rule of five (22), four important pharmacology-associated descriptors, including molecular weight (MW), the number of hydrogen bond donors (nHDon), the number of hydrogen bond acceptors (nHAcc) and octanol-water partition coefficients $(\mathrm{A} \log \mathrm{P})$, were calculated in order to evaluate the drug-likeness of HTTLF compounds.

Molecular docking. To determine whether HTTLF can interact with 17 key targets associated with RA $(23,24)$, molecular docking simulations were performed between HTTLF compounds and these targets by the LigandFit module of DS 2.0. Their protein crystal structures were retrieved from the Protein Data Bank (PDB; Table I) (25). All crystallographic waters were removed from the file and the hydrogen atoms were added. An inhibitor from the PDB file was used to define the active site. HTTLF compounds were docked onto the protein models. The interactions between these were evaluated using DockScore (26). The compounds with the top 20 DockScores were selected as potentially active compounds of HTTLF (13).

Network construction and analysis. Potentially active compounds and corresponding targets were analyzed using compound-target network (CTN) and target-disease network (TDN) (18). The CTN was constructed by linking the potential active compounds and their corresponding targets, and the TDN was constructed by linking the potential targets 
Table II. Statistics of key molecular properties of compounds in Huatan Tongluo Fang.

\begin{tabular}{|c|c|c|c|c|}
\hline Name & Mean & Standard Deviation & Minimum & Maximum \\
\hline Carbon count & 15.91 & 10.77 & 1.00 & 75.00 \\
\hline Nitrogen count & 0.38 & 0.92 & 0.00 & 5.00 \\
\hline Oxygen count & 3.50 & 5.53 & 0.00 & 47.00 \\
\hline Octanol-water partition coefficients & 3.36 & 4.16 & -4.62 & 18.62 \\
\hline Molecular weight & 278.85 & 203.10 & 53.06 & 1707.20 \\
\hline Number of rotatable bonds & 6.97 & 7.18 & 0.00 & 37.00 \\
\hline Number of hydrogen bond acceptors & 3.74 & 5.50 & 0.00 & 47.00 \\
\hline Number of hydrogen bond donors & 1.97 & 3.40 & 0.00 & 28.00 \\
\hline Molecular volume & 199.67 & 133.85 & 19.20 & 985.43 \\
\hline Molecular surface area & 292.18 & 187.51 & 72.33 & 1479.01 \\
\hline Molecular polar surface area & 66.23 & 94.03 & 0.00 & 812.37 \\
\hline JX & 2.54 & 0.68 & 0.97 & 4.70 \\
\hline JY & 2.62 & 0.70 & 1.02 & 4.72 \\
\hline Wiener & 2301.02 & 7270.50 & 9.00 & 88225.00 \\
\hline Zagreb & 94.71 & 82.67 & 10.00 & 682.00 \\
\hline
\end{tabular}

and their corresponding diseases. The associations between the targets and diseases were retrieved from the Therapeutic Targets Database (23). The above networks were generated and analyzed by Cytoscape version 2.8.3 (University of California, San Diego, CA, USA) (27).

\section{Results}

Molecular physicochemical property analysis of HTTLF. The distribution of the physicochemical properties of compounds from HTTLF was diverse (Table II). The majority of the compounds were observed to have clustered on the left side of the chemical space (Fig. 1). There was a large overlap between HTTLF compounds and drug/drug-like compounds in the chemical space. Fig. 2 presents the percentages of MW (<500), A $\log \mathrm{P}(<5)$, nHDon $(<5)$ and nHAcc $(<10)$ were $95.59,67.92,90.89$ and $89.31 \%$, respectively. According to the structure-activity relationship theory and Lipinski's rule of five $(22,28)$, the compounds from HTTLF possessed molecular diversity and drug-likeness.

Diverse functions of HTTLF. Docking results demonstrated that there were 127 potentially active compounds in HTTLF. The interactions between compounds and targets are presented in the compound-target network (CTN) (Fig. 3). The CNT showed that 50 compounds can interact with $\geq 2$ targets, while 77 compounds can interact with only one target (Fig. 4). The general network properties and key compounds in the CTN are listed in Tables III and IV, respectively. The values of network heterogeneity and network centralization were 1.336 and 0.108 , respectively. In addition, a TDN was constructed (Fig. 5). The TDN demonstrated that 17 targets were associated with 82 diseases (Table V) that belonged to 26 categories, such as musculoskeletal, immune system and cardiovascular diseases, and neoplasms. This suggests that HTTLF may demonstrate efficacy in targeting these diseases.
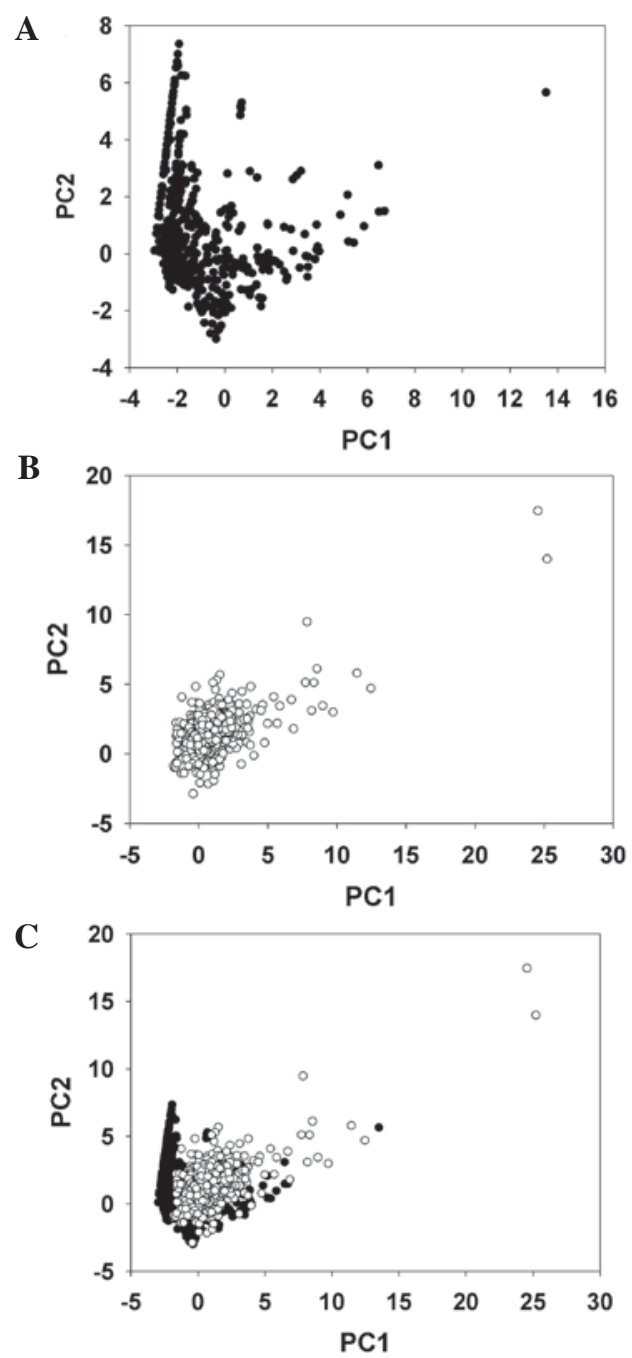

Figure 1. Chemical space distributions of compounds from HTTLF and the MDDR. (A) Chemical space distributions of compounds from HTTLF. (B) Chemical space distributions of compounds from MDDR. (C) Black circles represent compounds from HTTLF, whereas white circles represent drug/drug-like compounds from the MDDR. HTTLF, Huatan Tongluo Fang; PC, principal component; MDDR, MDL Drug Data Report. 
Table III. General network properties of the compound-target network.

\begin{tabular}{lc}
\hline Parameter & Compound-target network \\
\hline Number of nodes & 144 \\
Number of edges & 340 \\
Network density & 0.033 \\
Network heterogeneity & 1.336 \\
Isolated nodes & 0 \\
Number of self-loops & 0 \\
Multi-edge node pairs & 0 \\
Network centralization & 0.108 \\
Shortest paths & $20,592(100 \%)$ \\
Characteristic path length & 3.891 \\
Average number of neighbors & 4.722 \\
\hline
\end{tabular}

A

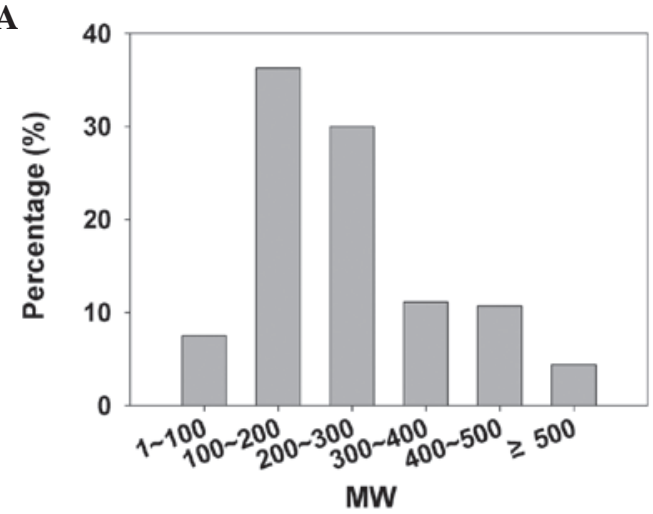

C

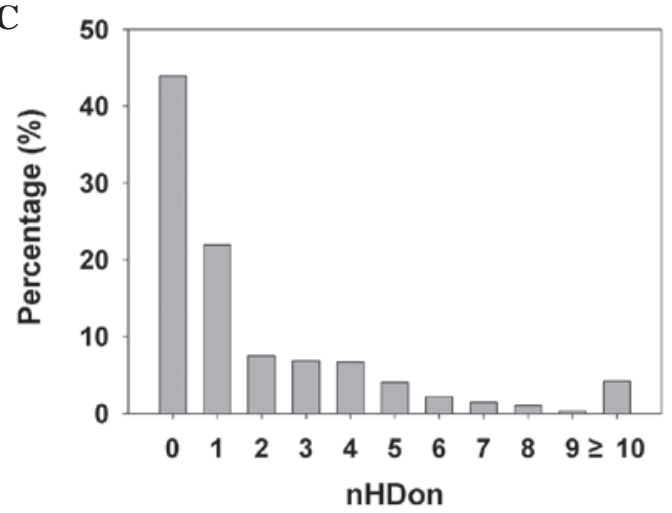

Table IV. Key compounds with top-10 degree in the compound-target network.

\begin{tabular}{|c|c|c|c|}
\hline Index & Degree & Chemical name & Known \\
\hline SP-27 & 12 & $\begin{array}{l}\text { Naringenin 7-O-beta- } \\
\text { D-glucoside }\end{array}$ & No \\
\hline BA-75 & 12 & Isoschaftoside & No \\
\hline BA-74 & 12 & Schaftoside & No \\
\hline BA-143 & 12 & Apigenin 6, 8-di-C-glucoside & No \\
\hline SP-68 & 11 & Chlorogenic acid & Yes \\
\hline SP-31 & 10 & Quercitrin & No \\
\hline SP-30 & 10 & Quercetin & Yes \\
\hline SP-1 & 10 & Amygdalin & Yes \\
\hline BA-54 & 10 & Deacetylcentapicrin & No \\
\hline BA-144 & 10 & Apioside & No \\
\hline
\end{tabular}

B

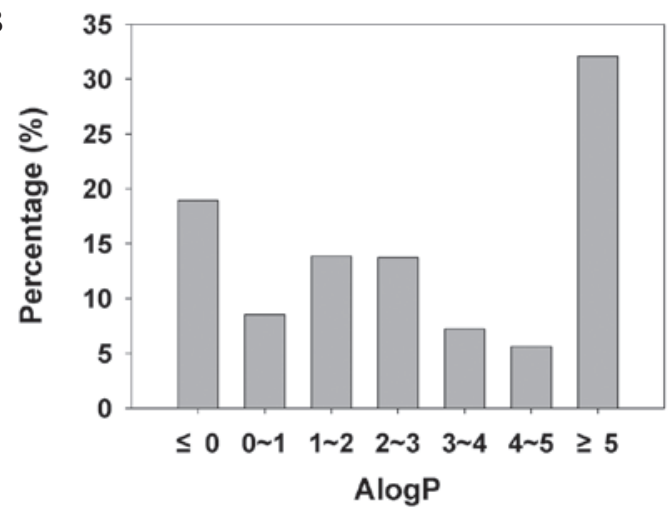

D

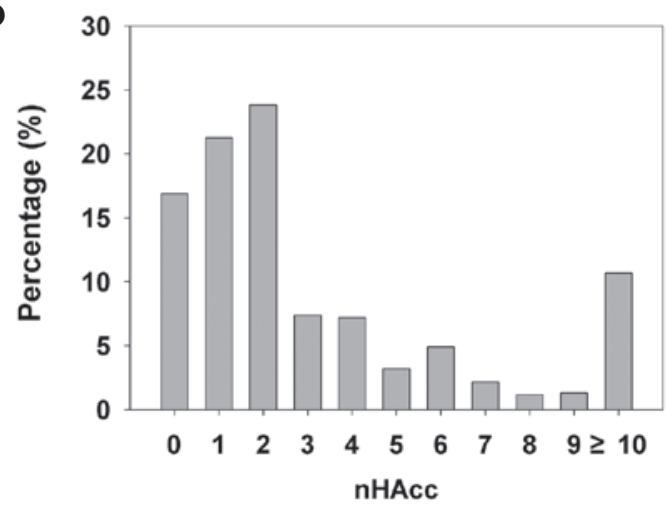

Figure 2. The distributions of four important molecular properties of compounds from Huatan Tongluo Fang. Molecular properties consist of (A) MW, (B) AlogP, (C) nHDon and (D) nHAcc. MW, molecular weight; AlogP, octanol-water partition coefficients; nHDon, number of hydrogen bond donors; nHAcc, number of hydrogen bond acceptors.

\section{Discussion}

RA is a common chronic inflammatory disease that results in a considerable burden for the patient and society. The cause of RA is not a single effect; it is caused by multiple molecular abnormalities (29). Numerous clinical studies have demonstrated that a number of Chinese herbal monomers, such as triptolide and sinomenine, have efficient therapeutic effects in treating RA $(30,31)$. It has been demonstrated that a combination of Tripterygium wilfordii polyglycoside and methotrexate can improve the therapeutic effects; in addition, the combination treatment can reduce side-effects and drug resistance (32). Furthermore, Tripterginum wilfordii polyglycoside in combination with glycyrrhizic acid has been demonstrated to reduce liver injury (33). Considering complex diseases, the model of drug discovery has been changed from identifying a single target to identifying multi-targets based on systems biology (34). Notably, herbal remedies 


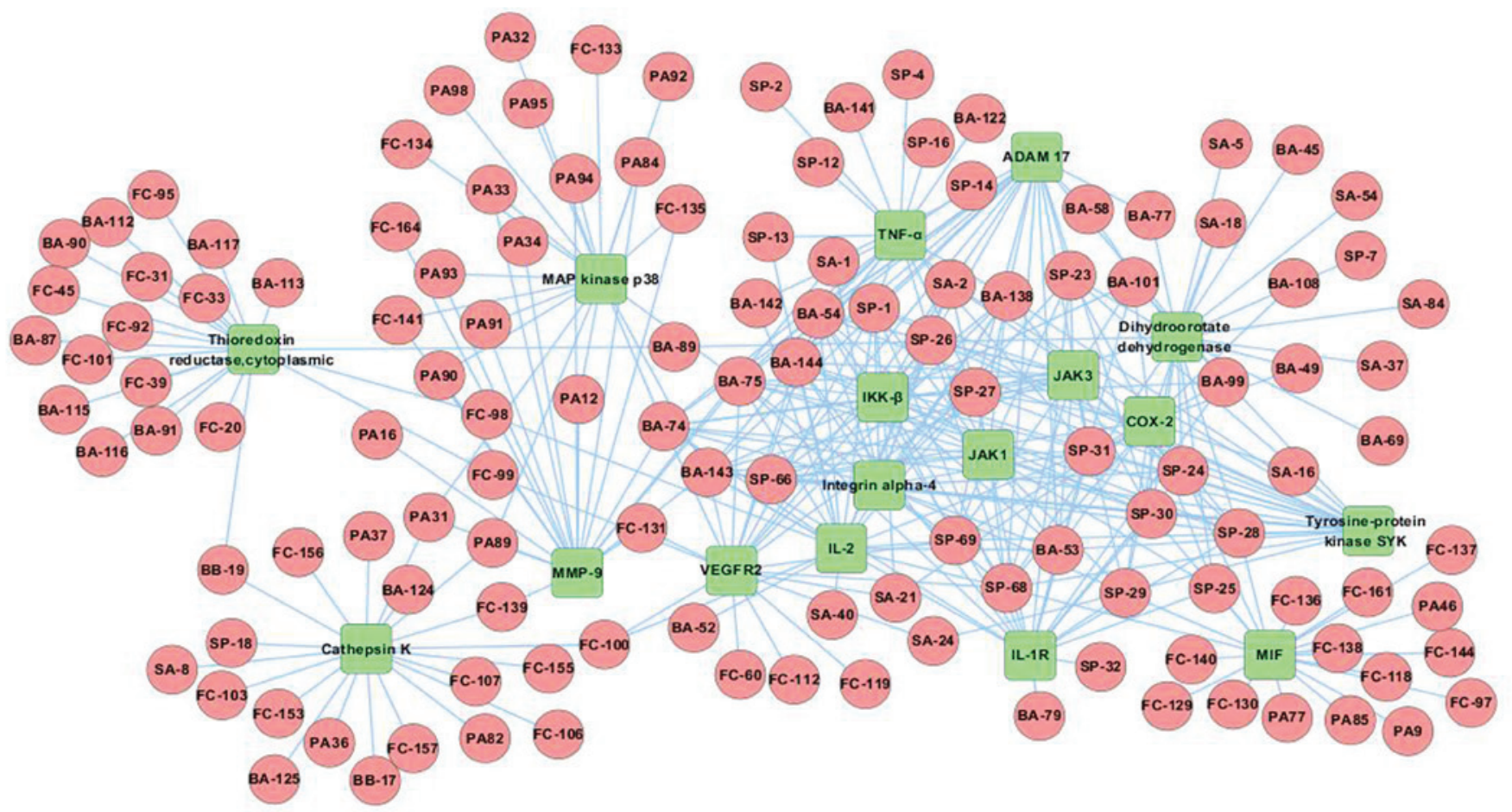

Figure 3. Compound-target network. Potentially active compounds are connected with associated diseases; the circles and rectangles represent the candidate compounds and target proteins, respectively. D, disease; BA, bile arisaema; SP, Semen persicae; FC, Flos carthami; SA, Sinapis alba; BB, Bombyx batryticatus; PA, Paeonia alba.

with the characteristics of multi-component and multi-target compounds are most prevalent and effective in the treatment of chronic illnesses in a number of Asian countries (35); thus, chemical components can be used effectively in Chinese and Western medicines. (36). Thus, it may be useful to study compounds, targets and networks to investigate how Chinese herbal ingredients are effective against RA.

HTTLF is a traditional herbal formula that has been widely used in the Xiamen Hospital of Traditional Chinese Medicine. In the present study, the results demonstrated that the chemical space distributions of compounds from HTTLF were diverse. The data also demonstrated that there is a large overlap between HTTLF compounds and drug/drug-like compounds in the chemical space. According to the QSAR, the compounds with similar chemical space have similar active properties (28). Thus, the compounds from HTTLF may have diverse properties; the majority of compounds possessed drug-like properties, which aids in identifying anti-RA compounds from HTTLF.

Docking results in the current study demonstrated that 127 compounds from HTTLF could interact with 17 targets associated with RA. Among them, 50 compounds had potentially a large number of drug properties, while 77 compounds had the potential to be used in combination therapy. These results demonstrate that HTTLF is a broad-spectrum herbal treatment. To further understand the association between potential compounds and their targets, a CTN was constructed. The network consisted of 144 nodes (127 compounds and 17 targets) and 340 edges. The compounds in the outer CTN displayed fewer interactions with targets than those in the

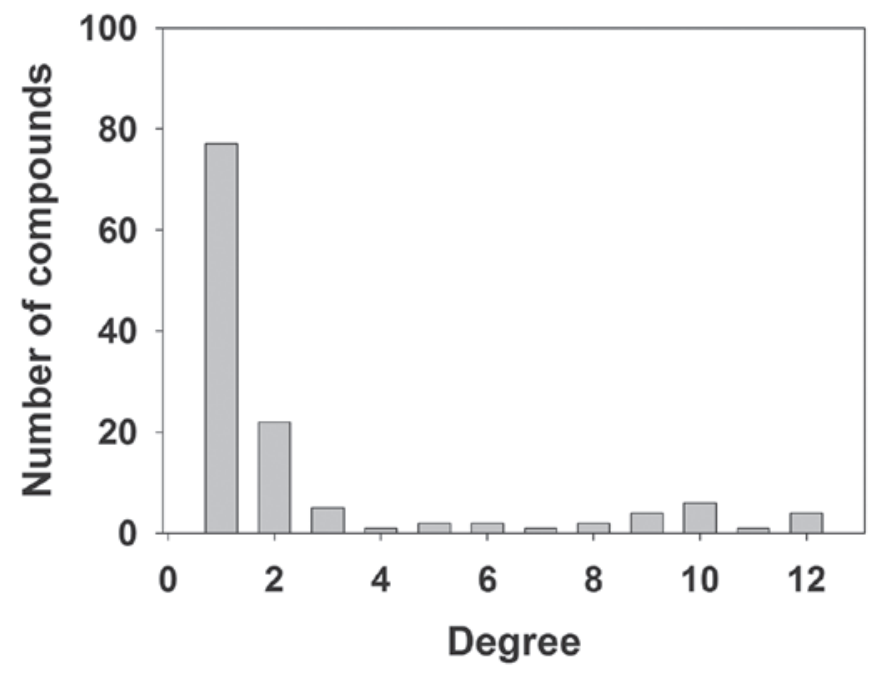

Figure 4. Degree distributions of compound nodes.

inner CTN. The values of network heterogeneity and network centralization were 1.336 and 0.108 , respectively. This indicated that a number of compounds were more central than others (37). For example, isoschaftoside (BA-75) had the largest number of target interactions, whereas $\beta$-carotene had one target interaction. Thus, HTTLF exhibited diverse therapeutic effects via interacting with the same or different targets.

Previous studies have demonstrated that particular compounds exhibit biological activities against targets associated with RA (38-41). For example, chlorogenic acid can 
Table V. Diseases associated with potential targets.

\begin{tabular}{|c|c|}
\hline Index & Disease \\
\hline D1 & Abdominal aortic aneurysm \\
\hline D2 & Acute pain \\
\hline D3 & Adenomatous polyposis \\
\hline D4 & Advanced lung cancer \\
\hline D5 & Allergic rhinitis, unspecified \\
\hline D6 & Allergy, unspecified \\
\hline D7 & Alzheimer's disease \\
\hline D8 & Amebiasis \\
\hline D9 & Arthritis \\
\hline D10 & Asthma \\
\hline D11 & Atherosclerosis \\
\hline D12 & Autoimmune diseases \\
\hline D13 & Bacterial infections \\
\hline D14 & Behcet's disease \\
\hline D15 & Bladder cancer \\
\hline D16 & Brain cancer \\
\hline D17 & Breast cancer \\
\hline D18 & Cancer, unspecific \\
\hline D19 & Cancers \\
\hline D20 & Carcinoma in situ, unspecified \\
\hline D21 & Carpal tunnel syndrome \\
\hline D22 & Chronic pain \\
\hline D23 & Colorectal cancer \\
\hline D24 & Congestive heart failure \\
\hline D25 & Coronary artery disease \\
\hline D26 & Diabetes mellitus \\
\hline D27 & Diabetic nephropathy \\
\hline D28 & Dysmenorrhea, unspecified \\
\hline D29 & Endometriosis \\
\hline D30 & Facial Pain \\
\hline D31 & Fibrosing alveolitis \\
\hline D32 & Genitourinary tumors \\
\hline D33 & Gestational hypertension \\
\hline D34 & Glomerulonephritis \\
\hline D35 & Graft-versus-host disease \\
\hline D36 & Guillain-barre syndrome \\
\hline D37 & Heart failure \\
\hline D38 & Hepatocellular carcinoma \\
\hline D39 & Hormone-refractory prostate cancer \\
\hline D40 & Human immunodeficiency virus disease \\
\hline D41 & Hyperimmunoglobulinemia D \\
\hline D42 & Inflammation \\
\hline D43 & Inflammatory bowel disease \\
\hline D44 & Kaposi's sarcoma \\
\hline D45 & Leprosy, unspecified \\
\hline D46 & Lung cancer \\
\hline D47 & Lymphoma, non-hodgkin \\
\hline D48 & Malaria \\
\hline D49 & Malignancies \\
\hline D50 & Malignant mesothelioma \\
\hline D51 & Meningioma \\
\hline
\end{tabular}

Table V. Continued.

\begin{tabular}{|c|c|}
\hline Index & Disease \\
\hline D52 & Multiple sclerosis \\
\hline D53 & Myocardial infarction \\
\hline D54 & Neurologic disorders \\
\hline D55 & Non-insulin-dependent diabetes mellitus \\
\hline D56 & Non-small cell lung cancer \\
\hline D57 & Obstructive airway disease \\
\hline D58 & Oropharyngeal squamous cell carcinoma \\
\hline D59 & Osteoarthritis \\
\hline D60 & Osteoporosis \\
\hline D61 & Pain, unspecified \\
\hline D62 & Pancreatic cancer \\
\hline D63 & Parasitic diseases \\
\hline D64 & Pathological angiogenesis \\
\hline D65 & Periodic fever syndrome \\
\hline D66 & Peutz-jeghers syndrome \\
\hline D67 & Pneumocystis infections \\
\hline D68 & Prostate cancer \\
\hline D69 & Psoriasis \\
\hline D70 & Renal cell carcinoma \\
\hline D71 & Restenosis \\
\hline D72 & Rheumatic diseases \\
\hline D73 & Rheumatoid arthritis \\
\hline D74 & Rheumatoid arthritis, unspecified \\
\hline D75 & Sepsis \\
\hline D76 & Septic shock \\
\hline D77 & Sjogren's syndrome \\
\hline D78 & Stroke \\
\hline D79 & Systemic lupus erythematosus \\
\hline D80 & Thrombocytopenia \\
\hline D81 & Tumors \\
\hline D82 & Ulcerative colitis \\
\hline
\end{tabular}

inhibit the expression of cyclooxygenase- 2 and attenuate pro-inflammatory cytokines (including interleukin- $1 \beta$ and tumor necrosis factor- $\alpha$ ), which may be beneficial for the prevention and treatment of inflammatory diseases. Quercetin and amygdalin have also been reported to possess anti-inflammation properties $(39,41)$. The combination of these three compounds may have synergistic actions on anti-inflammation. In addition, the CTN demonstrated that the three compounds shared 6 common targets. Thus, the synergistic actions of compounds in HTTLF may be responsible for the therapeutic efficacy of RA.

In order to further verify the diverse functions of HTTLF, a TDN was constructed. The network contained 17 targets and 82 diseases. For example, MMP-9 connected with 15 diseases, including advanced lung cancer, atherosclerosis, brain cancer, osteoarthritis and rheumatoid arthritis. Clinical studies have demonstrated that the expression of MMP-9 can reflect the progression of knee osteoarthritis, atherosclerotic coronary artery disease and RA (42-44). In addition, according to the Medical Subject Headings 


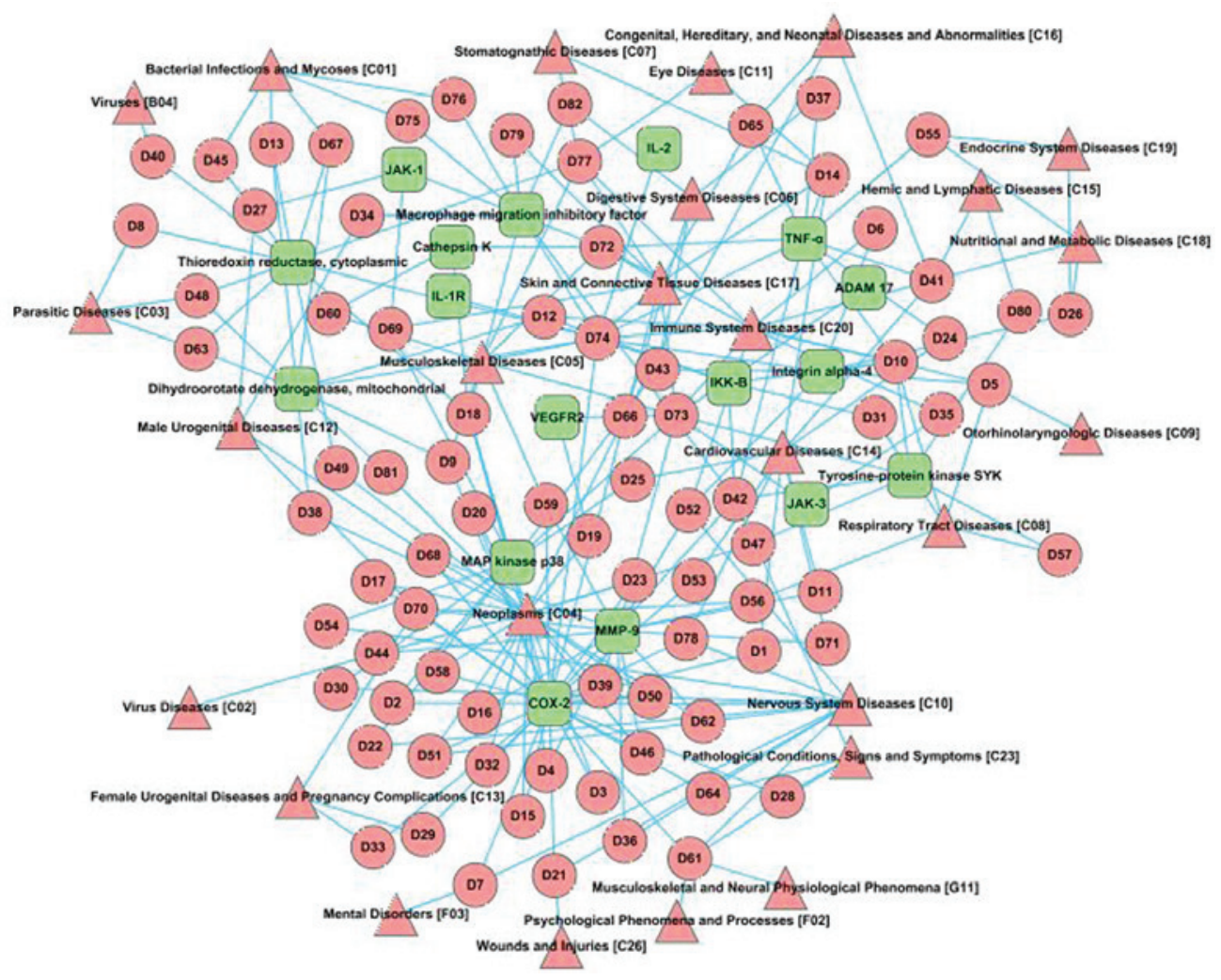

Figure 5. Target-disease network. In the target-disease network, 17 targets (rectangles) were associated with 82 diseases (circles) which belonged to 26 categories (triangles). D, disease.

(http://www.nlm.nih.gov/mesh/MBrowser.html) the 82 diseases were classified into 26 groups, including skin and connective tissue diseases, neoplasms, immune system diseases, cardiovascular diseases and musculoskeletal diseases. For example, osteoarthritis, osteoporosis and RA belong to musculoskeletal diseases; Sjögrkn's syndrome, asthma and allergic rhinitis are immune system diseases. This suggests that compounds from HTTLF have potential therapeutic effects on diverse diseases (45) in addition to RA; for example, amygdalin can be used to treat diseases such as asthma, tumors and diabetes (46). Thus, a TDN provides a visual representation of the association between potential active compounds of HTTLF and diseases through the targets associated with RA.

In conclusion, a novel platform of system pharmacology integrating physicochemical property analysis, active compound prediction, and compound-target and target-disease associate networks was created in the present study, in order to investigate the mechanisms underlying the therapeutic effects of HTTLF on RA. The results demonstrated that: i) Compounds from HTTLF exhibit diverse chemical space and drug-like properties; ii) a total of 127 compounds from HTTLF are regarded as potentially active compounds, interacting with 17 targets associated with RA; iii) compounds from HTTLF have diverse and synergistic actions in the treatment of RA, and exert therapeutic effects for other diseases, such as osteoarthritis, osteoporosis and neoplasms. This is consistent with the hypothesis of the 'same treatment for different diseases' in TCM. The present investigation provides a visual understanding of the chemical and pharmacological basis of TCM; this is beneficial to the discovery of anti-rheumatoid drugs from TCM. Based upon the present study, future research will include the extraction of effective constituents from HTTLF, which will be examined in vivo and in vitro for the discovery of anti-rheumatoid drugs from TCM.

\section{Acknowledgements}

The present study was supported by the National Natural Science Foundation of China (grant no. 81473574) and the Developmental Fund of ChenKeji Integrative Medicine (grant no. CKJ2015010).

\section{References}

1. Qin ZJ: The research progress of rheumatoid arthritis in diagnosis and treatment of Chinese and western medicines. Acta Med Sin 24: 224-227,2011. (In Chinese).

2. Fan FY and He XL: Research progress on traditional Chinese medicine for rheumatoid arthritis. Journal of Traditional Chinese Medicine University of Hunan 26: 60-61, 2006 (In Chinese)

3. Tong G, Zhang X, Tong W and Liu Y: Association between polymorphism in STAT4 gene and risk of rheumatoid arthritis: A meta-analysis. Hum Immunol 74: 586-592, 2013.

4. Xu J, Wang Q and Liu SY: Drug treatment of rheumatoid arthritis. Chin Community Doctors 17: 22-23, 2002. (In Chinese).

5. Niu LJ: Pharmacotherapy of rheumatoid arthritis. Pain Clin J 2: 106-109, 2006 (In Chinese).

6. Li QX: Research progress on immune mechanisms of traditional Chinese medicine for the treatment of rheumatoid arthritis. Research of Traditional Chinese Medicine 18: 53-55, 2002 (In Chinese).

7. Huang MC, Pai FT, Lin CC, Chang CM, Chang HH, Lee YC, Sun MF and Yen HR: Characteristics of traditional Chinese medicine use in patients with rheumatoid arthritis in Taiwan: A nationwide population-based study. J Ethnopharmacol 176 :9-16, 2015. 
8. Wang JM, Tao QW, Zhang YZ, Xu Y and Yan XP: Treating rheumatoid arthritis patients of Shen deficiency and cold invading syndrome by bushen quhan zhiwang decoction combined methotrexate: An evaluation of clinical efficacy and safety. Zhongguo Zhong Xi Yi Jie He Za Zhi 33: 614-618, 2013.(In Chinese).

9. Chen ZH: Clinical observation of Yishen Zhuanggu Tongbi Decoction in the Treatment of rheumatoid arthritis. Journal of Guangzhou University of Traditional Chinese Medicine 22: 19-22, 2005 (In Chinese).

10. Zhang YY, Chen JC, Qiu MS and Xu ZX: Clinical Observation of Huatan Tongluo Fang in the treatment of rheumatoid arthritis with phlegm-obstruction and blood-stasis Type. Traditional Chinese Medicine Journal 11: 49-52, 2012 (In Chinese).

11. Qiu MS, Chen JC, Zhang YY, Xu ZX, Jiang YF and Peng YF: Effect of Huatan Tongluo Fang on collagen-induced arthritis in rats. Journal of Beijing University of Traditional Chinese Medicine 35: 121-124, 2012 (In Chinese).

12. Zheng CS, Ye HZ, Xu XJ and Liu XX: Computational pharmacology study of tougu xiaotong granule in preventing and treating knee osteoarthritis. Chin J Integr Med 15: 371-376, 2009

13. Zheng CS, Xu XJ, Ye HZ, Wu GW, Li XH, Huang SP and Liu XX: Computational approaches for exploring the potential synergy and polypharmacology of Duhuo Jisheng Decoction in the therapy of osteoarthritis. Mol Med Rep 7: 1812-1818, 2013.

14. Shi SH, Cai YP, Cai XJ, Zheng XY, Cao DS, Ye FQ and Xiang Z: A network pharmacology approach to understanding the mechanisms of action of traditional medicine: Bushenhuoxue formula for treatment of chronic kidney disease. PLoS One 9: e89123, 2014.

15. Qiao X, Hou T, Zhang W, Guo S and Xu X: A 3D structure database of components from Chinese traditional medicinal herbs. J Chem Inf Comput Sci 42: 481-489, 2002.

16. Zhou JJ, Xie GR and Yang JX (eds): Handbook of the Chemical Constituents in Chinese Herb Original Plants. Chemical Industry Press, Beijing, pp1165-pp1211, 2004 (In Chinese).

17. Wang ZQ: Research progress of chemical composition and pharmacological effects of Rhizoma arisaematis. Drug Evaluation Research 32: 144-149, 2009 (In Chinese).

18. Zheng CS, Xu XJ, Ye HZ, Wu GW, Li XH, Xu HF and Liu XX Network pharmacology-based prediction of the multi-target capabilities of the compounds in Taohong Siwu decoction and their application in osteoarthritis. Exp Ther Med 6: 125-132, 2013.

19. Cheng SM, Wang HY, Li GY, Zhu Y, Zhao WB and Wang JH: Research progress of Bombyx batryticatus. Journal of Nongken Medicine 34: 443-448, 2012 (In Chinese).

20. Sheridan RP and Shpungin J: Calculating similarities between biological activities in the MDL Drug Data Report database. J Chem Inf Comput Sci 44: 727-740, 2004.

21. Hassan M, Bielawski JP, Hempel JC and Waldman M: Optimization and visualization of molecular diversity of combinatorial libraries. Mol Divers 2: 64-74, 1996.

22. Lipinski CA, Lombardo F, Dominy BW and Feeney PJ: Experimental and computational approaches to estimate solubility and permeability in drug discovery and development settings. Adv Drug Deliv Rev 46: 3-26, 2001.

23. Zhu F, Shi Z, Qin C, Tao L, Liu X, Xu F, Zhang L, Song Y, Liu X, Zhang J, et al: Therapeutic target database update 2012: A resource for facilitating target-oriented drug discovery. Nucleic Acids Res 40: D1128-D1136, 2012

24. Hashimoto N, Iwasaki T, Kitano M, Ogata A and Hamano T: Levels of vascular endothelial growth factor and hepatocyte growth factor in sera of patients with rheumatic diseases. Mod Rheumatol 3: 129-134, 2003

25. Rose PW,Prlić A, BiC, Bluhm WF, Christie CH, Dutta S, Green RK, Goodsell DS, Westbrook JD, Woo J, et al: The RCSB Protein Data Bank: Views of structural biology for basic and applied research and education. Nucleic Acids Res 43 (D1): D345-D356, 2015.

26. Montes M, Braud E, Miteva MA, Goddard ML, Mondésert O, Kolb S, Brun MP, Ducommun B, Garbay C and Villoutreix BO Receptor-based virtual ligand screening for the identification of novel CDC25 phosphatase inhibitors. J Chem Inf Model 48: 157-165, 2008 .
27. Smoot ME, Ono K, Ruscheinski J, Wang PL and Ideker T: Cytoscape 2.8: New features for data integration and network visualization. Bioinformatics 27: 431-432, 2011.

28. Dobson CM: Chemical space and biology. Nature 432: 824-828, 2004.

29. Tanaka Y: Current concepts in the management of rheumatoid arthritis. Korean J Intern Med 31: 210-218, 2016.

30. Wang NN, Wang AW and Hao QX: New progress of study on traditional Chinese medicine for the treatment of rheumatoid arthritis. Modern Journal of Integrated Chinese Traditional and Western Medicine 20: 2334-2336, 2011 (In Chinese).

31. Zheng CE: The experience of research of integrated traditional Chinese and Western medicine for the treatment of rheumatoid arthritis. Integrated Traditional Chinese and Western Medicine 4: 255-256, 2012 (In Chinese)

32. Wu YJ, Lao ZY and Zhang ZL: Clinical observation on small doses Tripterygium wilfordii polyglycoside combined with methotrexate in treating rheumatoid arthritis. Zhongguo Zhong Xi Yi Jie He Za Zhi 21: 895-896, 2001. (In Chinese).

33. Li YS,Yang JH,Fan SY and Zhang YY:The experimental study of Tripterygium wilfordii polyglycoside combination therapy with glycyrrhizic acid for rheumatoid arthritis on efficacy enhancing and toxicity reducing. Chin J Tradit Med Sci Technol 15:293, 2008. (In Chinese).

34. Kitano H: A robustness-based approach to systems-oriented drug design. Nat Rev Drug Discov 6: 202-210, 2007.

35. Chen M, Feng W, Cao H, Zou L, Chen C, Batrup A, Nielsen AB, Li H, Kassem M, Zou X and Bünger C: A traditional Chinese medicine formula extracts stimulate proliferation and inhibit mineralization of human mesenchymal stem cells in vitro. J Ethnopharmacol 125: 75-82, 2009.

36. Li XD, Huang Q and Xu XJ: Target-Li gand space distribution of the compound sets from Xuefu Zhuyu Decoction, a traditional chinese medicinal recipe. Acta Physico-Chimica Sinica 24: 547-550, 2008 (In Chinese).

37. Ma JL, An LL and Xing X: Topology analysis on urban public traffic networks. Journal of Shanxi Teachers University 23: 51-55, 2009 (In Chinese)

38. Yang B, Qiu Y, Wang LP and Zhang XL: Studies on the anti-inflammatory molecular mechanism of chlorogenic acid extracted from Lonicera confusa DC in vitro. Chinese Pharmacological Bulletin 25: 542-545, 2009 (In Chinese).

39. Huang JQ, Sun WJ, Wang SW and Zhu MZ: Studies on the anti-inflammatory and antioxidant activity of quercetin in rats with gouty arthritis. China Journal of Experiment Traditional Medical Formulae 18: 169-173, 2012 (In Chinese).

40. Wang MY, Wei YF and Li XB: Chemical constituents of antirheumatism fraction from Urtica fissa. Chinese Traditional and Herbal Drugs 37: 1300-1303, 2006 (In Chinese).

41. Lv JZ and Deng JG: Research progress in pharmacological effects of amygdalin. Drugs \& Clinic 27: 530-535, 2012 (In Chinese)

42. He ZK and Shen JW: The value of MMP-2, MMP-3, MMP-9 and TIMP-1 levels in the evaluation of knee joint osteoarthritis Chongqing Medical Journal 42: 3872-3874, 2013 (In Chinese).

43. Wu ZB, Lu N, Wang YH, Shi ZG, Fan CM, Jia J, Liu L, Leng N and Zhu P: Association of serum MMP-2 and MMP-9 and progression of joint damage in early rheumatoid arthritis. Chinese Journal of Immunology 22: 260-262, 2006 (In Chinese).

44. Gao F, Chen LL, Yan SR, Guo SR, Zhao LH, Yang P, Zhang CW and Ji XB: Correlation between serum levels of MMP-1, MMP-9, TIMP-1, IL-6 and the severity of atherosclerotic coronary artery disease. Journal of Clinical Research 30: 2384-2386, 2013 (In Chinese).

45. Royall J and Lyon B: Sea-change or change challenge? Health information access in developing countries: The U.S. National Library of Medicine experience. Afr Health Sci 11:457-463,2011.

46. Zhou C, Qian L, Ma H, Yu X, Zhang Y, Qu W, Zhang X and Xia W: Enhancement of amygdalin activated with $\beta$-D-glucosidase on HepG2 cells proliferation and apoptosis. Carbohydr Polym 90: 516-523, 2012. 